\title{
Hypoglycemic and Hypolipidemic Effects of Cucumber, White Pumpkin and Ridge Gourd in Alloxan Induced Diabetic Rats
}

\author{
R. Sharmin ${ }^{1}$, M. R. I. Khan ${ }^{2}$, Most. A. Akhter ${ }^{2 *}$, A. Alim ${ }^{3}$, M. A. Islam², \\ A. S. M. Anisuzzaman ${ }^{2}$, and M. Ahmed ${ }^{2}$ \\ ${ }^{1}$ Department of Pharmacy, Jessore Science and Technology University, Jessore \\ ${ }^{2}$ Department of Pharmacy, Rajshahi University, Rajshahi-6505, Bangladesh \\ ${ }^{3}$ Square Pharmaceuticals Ltd., Kaliakoir, Gazipur-1750, Dhaka, Bangladesh
}

Received 2 April 2012, accepted in final revised form 20 December 2012

\begin{abstract}
Ethanolic extracts of some fruits of Cucurbitaceae family such as Cucumis sativus (cucumber), Lagenaria siceraria (white pumpkin), Luffa acutangula (ridge gourd), Benincasa hispida (ash gourd), Citrullus lanatus (sweet melon) and Cucarbita maxima (pumpkin) have been studied for their hypoglycemic effects on alloxan induced diabetic rats (AIDRs). Screening results suggested that among the tested fruits the hypoglycemic potency follows: cucumber > white pumpkin > ridge gourd. These three fruit-extracts were further investigated for their hypoglycemic, hypolipidemic and glycogenesis effects. Cucumber, white pumpkin and ridge gourd extracts reduced blood glucose level by 67,65 and 51\%, respectively at 12 hours after single intraperitoneal injection; while reduced the low density lipoprotein (LDL) level to 13, 28 and 86\%, respectively in AIDRs. The maximum reduction $87 \%$ was observed by cucumber extract. Cucumber, white pumpkin and ridge gourd extracts reduced total cholesterol level to 29,15 and $38 \%$, respectively comparing with the diabetic control group. Here the maximum reduction of $85 \%$ was observed by white pumpkin extract. Cucumber, white pumpkin and ridge gourd also reduced triglyceride levels to 72,68 and $80 \%$, respectively. Maximum reduction of $32 \%$ was observed by white pumpkin. Significant improvement of glycogenesis was also observed by ridge gourd extracts in AIDRs.
\end{abstract}

Keywords: Blood glucose; Hypoglycemic; Hypolipidemic; Diabetic rats.

(C) 2013 JSR Publications. ISSN: 2070-0237 (Print); 2070-0245 (Online). All rights reserved.

doi: http://dx.doi.org/10.3329/jsr.v5i1.10252 J. Sci. Res. 5 (1), 161-170 (2013)

\section{Introduction}

Diabetes is a growing health problem in the world today. Almost $1.3 \%$ of the population suffers from this disease throughout the world [1] and number of diabetics is increasing by $6 \%$ per year [2].

\footnotetext{
*Corresponding author: afia2441139@yahoo.com, aashuva@yahoo.com
} 
Diabetes mellitus is a metabolic disorder characterized by chronic hyperglycemia resulting from defects in insulin secretion, insulin action, or both. It is associated with reduced life expectancy, significant morbidity due to specific diabetes related micro vascular complications (retinopathy, nephropathy and neuropathy), increased risk of macrovascular complications (ischemic heart disease, stroke and peripheral vascular disease) and diminished quality of life [3]. The major mode of controlling diabetes can be achieved by diet, exercise, and insulin replacement therapy and/or by different oral hypoglycemic drugs. In modern medical system, managing diabetes without side effects is still a challenge.

Unfortunately, after the introduction of sulfonylurea and metformin about 50 years back no major lead has been obtained in this direction of finding a proper drug for diabetes. Plant materials, which are being used as traditional medicine for the treatment of diabetes, are considered one of the good sources for a new drug or a lead to make a new drug. Plant extract or different folk plant preparations are being prescribed by the traditional practitioners and also accepted by the users for diabetes in many countries especially in third world countries. Now-a days more than 400 plants are being used in different forms for hypoglycemic effects all the claims practitioners or users are neither baseless nor absolutely. Therefore, a proper scientific evaluation, a screening of plant by pharmacological tests followed by chemical investigations is necessary [4]. Now a days, scientists and researchers are very much interested on research of natural plant products all over the world and a large amount of substantiation have shown the immense potential of medicinal plants used traditionally [5]. In the last few years there has been an exponential growth in the field of herbal medicine and these drugs are gaining popularity both in developing and developed countries because of their natural origin and less side effects [6]. Cucurbits (Cucurbitaceae) are among the most important plant families supplying humans with edible products and useful fibers. There are several plants of Cucurbitaceae family, which are established for their antidiabetic properties such as Momordica charantia [7], and Coccinia indica [8]. Our preliminary screening results suggested that Cucumis sativus (cucumber), Luffa acutangula (ridge gourd) and Lagenaria siceraria (white pumpkin) had more potent antidiabetic effects. Therefore, in this work, we tried to investigate the antidiabetic and hypolipidemic effects of these three fruits extracts of Cucurbitaceae family in chemically induced diabetic rats model.

Cucumber (Cucumis sativus) originated in India but soon cultivated in different parts of the world. In Mexico, cucumber is one of the edible plants with hypoglycemic activity. Antihyperglycemic effect of this plant was studied in healthy rabbits, which significantly decreased the area under the glucose tolerance curve and the hyperglycemic peak [9].

Ridge gourd (Luffa acutangula) is believed to have originated in India, but has now spread pantropically to all areas with a high rainfall. In southern and eastern Asia it is a widely cultivated vegetable. Ridge gourd is a demulcent and diuretic and seeds are emetic and purgative [10]. The analgesic and hypoglycemic activities of ridge gourd was evaluated in Swiss Webster mice where methanol extracts of ridge gourd showed significant hypoglycemic activities when administered 15 min after glucose load using a modified oral glucose tolerance test [11]. However, hypolipidemic and hepatoprotective 
activities of cucumber (Cucumis sativus) and ridge gourd (Luffa acutangula) have not been investigated so far.

White pumpkin (Lagenaria siceraria) is indigenous to Africa and that it reached temperate and tropical areas in Asia and the Americas. It is a fruit vegetable used throughout Bangladesh and India. Petroleum ether fraction of white pumpkin is reported to possess antioxidant activity and hypolipidemic activity [12]. However there is a lacking of enough evidence about the hypoglycemic activity of white pumpkin and its hepatoprotective activities have not yet been investigated.

\section{Materials and Methods}

\subsection{Plant materials}

The fresh fruits of cucumber, white pumpkin, ridge gourd, ash gourd, sweet melon and pumpkin were collected from the local market and authenticated by botanist Dr. A. H. M. Mahbubur Rahman (Department of Botany, University of Rajshahi). They were cut into small pieces, dried completely under the mild sun and ground with an electric grinder into coarse powder. The powder was used for cold extraction.

\subsection{Preparation of crude extracts}

The coarse powders from different fruits were soaked in $95 \%$ ethanol for 7 days and were kept at room temperature with occasional shaking and stirring. When the solvent became concentrated, the liquid alcohol contents were filtered through cotton and then through filter paper (Whatman filter paper no. 1). The ethanolic solution was allowed to evaporate using rotary evaporator. Thus the highly concentrated ethanol extracts were obtained which were further dried completely under mild sun and by freeze-drying. The dried extracts were then preserved in the refrigerator for the experimental use.

\subsection{Drugs and chemicals}

Compounds were purchased from commercial sources as follows: Alloxan monohydrate; Loba Chemiie, Mumbai, India. Total cholesterol (TC) and triglyceride (TG) kits; Boehringer Mannheim, GmbH, Germany. Serum LDL diagnostic kits; Crescent Diagnostics, Jeddah. Glycogen test diagnostic kit was o-toluidine reagent. The active drug, metformin hydrochloride was the generous gift from Square Pharmaceuticals Ltd. Pabna Bangladesh.

\subsection{Induction of diabetes}

Long-Evans female rats weighing about 110 -120 g were purchased from Animal House of International Centre for Diarrheal Disease Research, Bangladesh (ICDDR, B). Prior to the commencement of experiment, the rats were acclimatized in a well-ventilated animal house at $25^{\circ} \mathrm{C}$ for a period of one week with adequate food and water ad libitum. For the 
development of diabetic model, rats were grouped into six groups. Each group contains three rats. After overnight fasting, a freshly prepared solution of alloxan monohydrate $(120 \mathrm{mg} / \mathrm{kg}$ body weight in normal saline) was administered intraperitoneally into group II-VI. Group I kept as normal control group that did not receive the chemical. After 48 hours their blood glucose content was measured by glucometer (Clever Check, Germany) according to the manufacturer's protocol. Rats with blood glucose levels above 11.1 $\mathrm{mM} / \mathrm{L}$ were selected for the study [13]. Their base line blood glucose level was also measured just prior to the administration of alloxan.

\subsection{Treatment of the animal}

Group I and II served as non-diabetic and diabetic control group, respectively. Group III stands for metformin control group in which metformin was administered as a single intraperitoneal dose of $150 \mathrm{mg} / \mathrm{kg}$ body weight. Group IV, V and VI received cucumber, white pumpkin and ridge gourd extracts, respectively as a single intraperitoneal dose of $200 \mathrm{mg} / \mathrm{kg}$ body weight. The blood samples were analyzed for blood glucose content at 0 , 4,8 , and 12 hours, respectively.

\subsection{Determination of serum total cholesterol (TC), serum triglyceride (TG) and serum low density lipoprotein (LDL) levels}

After completion of 12 hours experimental period rats were sacrificed and approximately 3-5 ml of blood samples were collected directly from heart by syringes. The collected blood samples were centrifuged at $4000 \mathrm{rpm}$ for 10 minutes and the resulting supernatant was obtained as serum. Serum TC, TG and LDL concentrations were analyzed by UV spectrophotometric method (Shimadzu UV-1200, Tokyo, Japan) using wet reagent diagnostic kits according to the manufacturer's protocol.

\subsection{Estimation of glycogen content in liver}

The liver tissues collected from sacrificed rats were analyzed for glycogen content by UV spectrophotometric method using o-toluidine reagent diagnostic kits as we have reported previously [14]. Briefly, this test utilizes the o-toluidine-glucose-coupling reaction for the estimation of glycogen after trichloroacetic acid (TCA) extraction, precipitation by alcohol and hydrolysis.

\subsection{Statistical analysis}

Data were expressed as mean \pm standard error of mean (SEM). Statistical comparisons were performed by one-way analysis of variance (ANOVA), or students paired or unpaired $t$-test where appropriate. Results are considered to be significant when $p$ values 
were less than $0.05(p<0.05)$. Statistical calculations and the graphs were prepared using Graph Pad Prism version 5.00 for Windows (Graph Pad Software, San Diego, CA, USA).

\section{Results}

\subsection{Effect of cucumber, white pumpkin, and ridge gourd extracts on fasting blood glucose (FBG) level in alloxan induced diabetic rats (AIDRs)}

After single intraperitoneal injection of cucumber, white pumpkin, and ridge gourd extracts $(200 \mathrm{mg} / \mathrm{kg}$ body weight), their fasting blood glucose (FBG) levels were measured at $0,4,8$, and $12 \mathrm{hrs,}$, respectively. The cucumber extracts reduced FBG level to $81.02,58.65$ and $32.61 \%$ at 4,8 and $12 \mathrm{hrs}$, respectively. Whereas, white pumpkin reduced FBG level to $85.12,58.82$ and $34.60 \%$; ridge gourd $85.73,67.75$ and $48.89 \%$ and metformin $\mathrm{HCl}$ to $69.20,44.95$ and $25.44 \%$ at 4,8 and $12 \mathrm{hrs,} \mathrm{respectively.} \mathrm{In} \mathrm{each} \mathrm{case}$ the effects were significantly different $(P<0.05)$ at 8 and $12 \mathrm{hrs}$ from the diabetic control group and maximum reduction of FBG level was achieved at $12 \mathrm{hr}$ by $67.38,65.39,51.10$ and $69.20 \%$ for cucumber, white pumpkin, ridge gourd and metformin $\mathrm{HCl}$, respectively. The results are shown in Fig. 1.

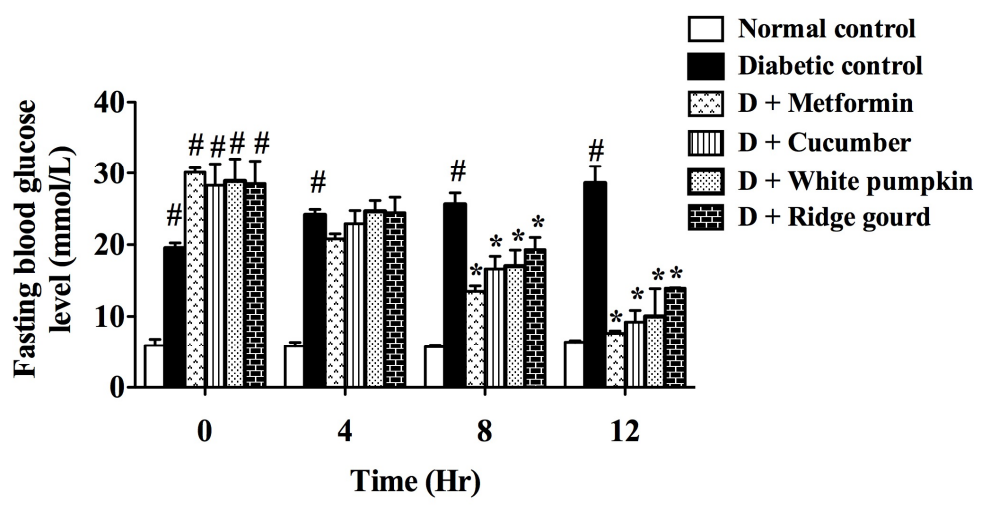

Fig. 1. Effect of cucumber, white pumpkin, and ridge gourd on FBG levels in AIDRs. \# indicates significant changes (increase) of blood glucose level compared with normal control group. * indicates significant changes (decrease) of FBG level in diabetic rats after treatment compared with zero $\mathrm{hr}$ treatment group. The results are expressed as means \pm SEM.

3.2. Effect of cucumber, white pumpkin and ridge gourd on total cholesterol (TC), triglyceride (TG) and low-density lipoprotein (LDL) levels in AIDRs

After induction of diabetes, the TC, TG and LDL levels were increased significantly in AIDRs. Cucumber, white pumpkin, ridge gourd and metformin $\mathrm{HCl}$ decreased the 
elevated serum TC levels to $28.83,14.91,38.38$ and $14.50 \%$, respectively. The maximum reduction of $85.08 \%$ was observed by white pumpkin extract. The elevated serum TG levels were reduced to $70.64,68.24,79.64$ and $60.48 \%$, by cucumber, white pumpkin, ridge gourd and metformin $\mathrm{HCl}$, respectively. The maximum reduction of $31.76 \%$ was observed by white pumpkin. On the other hand, serum LDL level was reduced to 13.07, $27.67,85.66$ and $16.05 \%$ when treated with cucumber, white pumpkin, ridge gourd and metformin $\mathrm{HCl}$, respectively. A maximum reduction of $86.92 \%$ was observed by cucumber ethanol extract. The summarized results are shown in the Figs. 2, 3 and 4 for TC, TG, and LDL levels, respectively.

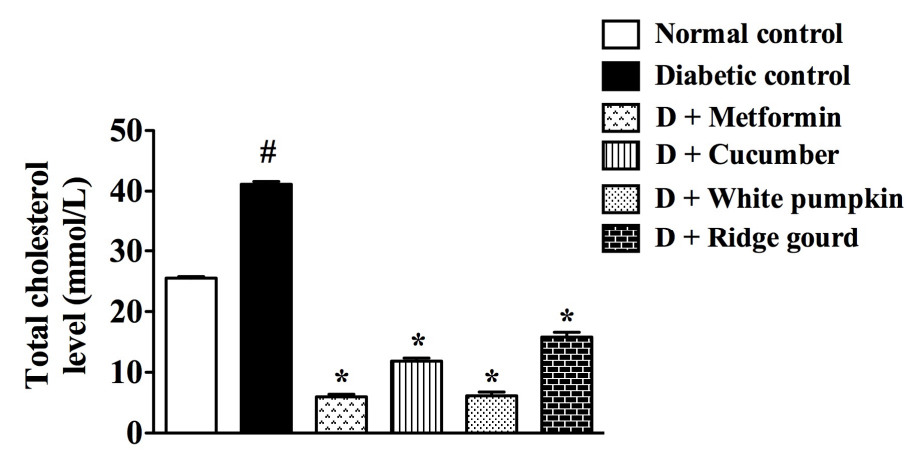

Fig. 2. Effect of cucumber, white pumpkin and ridge gourd on TC levels in AIDRs. ${ }^{\text {indicates }}$ significant difference from normal control after diabetes induction. * indicates significant change from diabetic control group after treatment with different extracts.

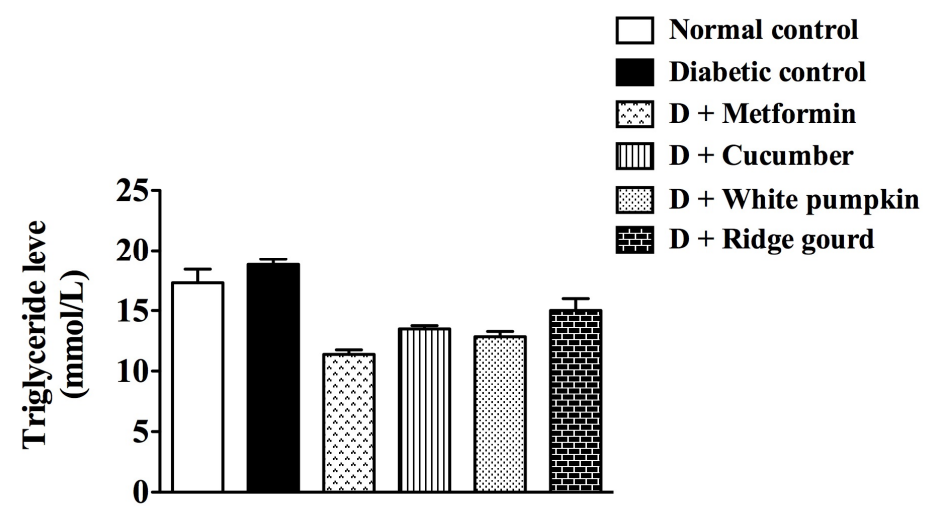

Fig. 3. Effect of cucumber, white pumpkin and ridge gourd on TG levels in AIDRs. After treatment with the extracts of cucumber, white pumpkin and ridge gourd, there was a tendency of the decrease of elevated TG levels. However, the reduction was not significantly different. 


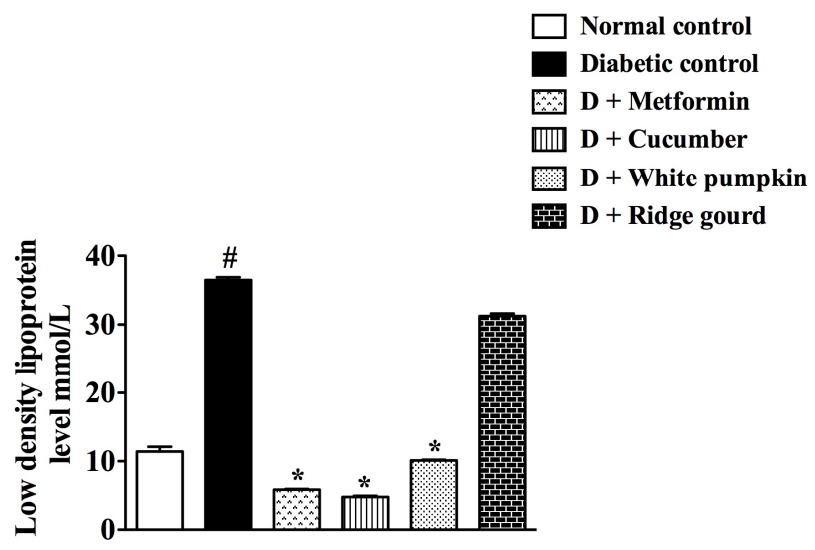

Fig. 4. Effect of cucumber, white pumpkin and ridge gourd on LDL levels in AIDRs. " indicates significant difference from normal control after diabetes induction. * indicates significant change from diabetic control group after treatment with different extracts.

\subsection{Effect of cucumber, white pumpkin, and ridge gourd on glycogen synthesis in} AIDRs

In this study it was found that the level of glycogen content in liver was reduced to $75.32 \%$ in diabetic rats as compared to the normal control group. Treatment of diabetic rats with metformin standard, cucumber, and ridge gourd extracts improved the glycogen levels to $196.10 \%, 107.79 \%$ and $149.35 \%$, respectively as shown in the Fig. 5 and Table 1.

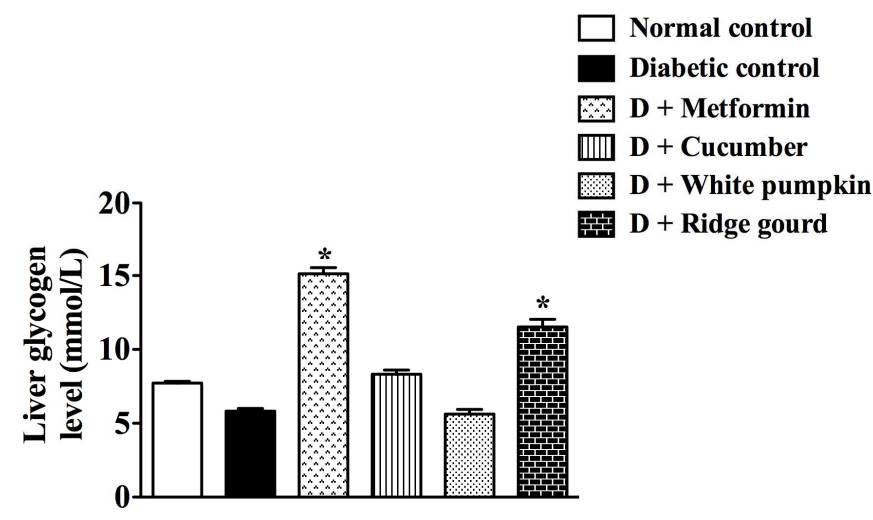

Fig. 5. Effect of cucumber, white pumpkin, and ridge gourd on glycogen synthesis in AIDRs. '*' indicates significant improvement of glycogen levels after treatment compared with diabetic control group. 
Table 1. Effect of cucumber, white pumpkin, and ridge gourd on glycogen synthesis in AIDRs. '*' indicates significant improvement of glycogen levels after treatment compared with diabetic control group.

\begin{tabular}{lc}
\hline \multicolumn{1}{c}{ Treatment } & $12 \mathrm{hr}$ \\
\hline Normal control & $7.7 \pm 0.01$ \\
Diabetic control & $5.8 \pm 0.01$ \\
D+metformin & $15.1 \pm 0.04^{*}$ \\
D+cucumber & $8.3 \pm 0.02$ \\
D+white pumpkin & $5.6 \pm 0.03$ \\
D+ridge gourd & $11.5 \pm 0.05^{*}$ \\
\hline
\end{tabular}

Values are expressed as mean \pm SEM of 3 experiments.

\section{Discussion}

Numerous oral hypoglycemic drugs exist alongside insulin; still there is no promising therapy to cure diabetes [15]. In recent years, many traditional medicinal plants were tested for their antidiabetic potential in the experimental animals [16]. Although some of these plants have great reputation in the indigenous system of medicine for their antidiabetic activities, many remain to be scientifically established.

In the light of the literature of the Cucurbitaceae family, we made an attempt to study the effect of cucumber (Cucumis sativus), white pumpkin (Lagenaria siceraria) and ridge gourd (Luffa acutangula) ethanolic extracts in AIDRs to evaluate their hypoglycemic, hypolipidemic and glycogenesis effect.

In this work we have shown that cucumber, white pumpkin and ridge gourd has significantly decreased the elevated blood glucose levels in AIDRs (Fig. 1). The possible mechanism by which these fruits extracts bring about their antidiabetic action may be by potentiating the insulin effect of plasma by stimulating insulin release from the remnant pancreatic $\beta$-cells or its release from the bound form [17]. Beside this, it might involve an extra-pancreatic action in these alloxan-diabetic rats, which might include the stimulation of peripheral glucose utilization or enhancing glycolytic and glycogenic processes with concomitant decrease in glycogenolysis and gluconeogenesis [18]. The antihyperglycemic activity of cucumber, white pumpkin and ridge gourd may also be due to the presence of hypoglycemic saponins, tannins, triterpines, alkaloids, flavonoids etc [19].

Hypercholesterolemia and hypertriglyceridemia have been reported to occur in diabetic rats [20-22]. Our previous work also reported hypertriglyceridemia and hyperlipidemia in AIDRs [14]. The ethanol extracts from cucumber, white pumpkin and ridge gourd significantly decreased serum cholesterol level in AIDRs (Fig. 2). Again cucumber and white pumpkin significantly decreased serum LDL level (Fig. 4). The 
mechanism of hypocholesterolemic action of cucumber, white pumpkin and ridge gourd and LDL lowering action of these extracts are not known; it could be mediated through the control of tissue metabolism and improved insulin secretion and action.

In the present study, we have also showed that the fruits extracts restored the depressed hepatic glycogen levels (Fig. 5 and Table 1). Our results showed that supplementation of diabetic rats with ethanol extracts of cucumber and ridge gourd resulted in significant elevation in hepatic glycogen content. Decreased activities of enzymes like glucose-6-phosphate and hexokinase are involved in glucose homeostasis in liver and kidney resulting in depletion of liver and muscle glycogen content [23]. Treatment with these fruits extract might increase the activity of the said enzymes to the control level indicating an overall increase in glucose influx. The exact mechanism of action needs further investigation.

\section{Conclusions}

From the current study, it is concluded that the ethanol extracts of Cucurbitaceae family fruits, cucumber, white pumpkin and ridge gourd has significant antihyperglycemic effects in AIDRs. They also have the capacity to reduce the elevated lipid profiles in AIDRs. Ridge gourd has also significant effects to restore the depressed hepatic glycogen levels in AIDRs. Therefore, we believe that these fruits extracts can be useful, at least as an adjunct, in the therapy of diabetes, a condition in which hyperglycemia and hyperlipidemia coexist quite often. However, further study is necessary for the screening of chemical compounds and the structure elucidation of the respective antidiabetic leads as well as their exact mechanism.

\section{Acknowledgements}

We thank Pharmacy Department of Rajshahi University for providing necessary facilities to carry out this research work. The present work was supported by the National Science and Information and Communication Technology (NSICT), Dhaka, Bangladesh for financial assistance to the first author and the authors would like to extend their gratitude to the Director, Animal Research Centre, ICDDR,B for providing necessary facilities.

\section{References}

1. R. Ghosh, K. H. Sharatchandra, S. Rita and I. S. Thokchom, Indian J Pharmacol. 36 (4), 222 (2004).

2. C. R. Resmi, A. Fathima, B. Sinilal and M. S. Latha, Indian drugs 38 (6), 319 (2001).

3. Definition and diagnosis of diabetes mellitus (WHO and IDF, Geneva, Switzerland, 2006) p. 5.

4. N. Nahar, Traditional medicine, $18^{\text {th }}$ Ed. (OBH Publishing Co. Pvt. Ltd., New Delhi, 1993) pp. 205-209.

5. K. M. Ali, K. Chatterjee, D. De, T. K. Bera and D. Ghosh, Int. J. Applied Res. in Natural Products 2 (3), 13 (2009).

6. M. Modak, P. Dixit, J. Londhe, S. Ghaskadbi and T. P. A. Devasagayam, J. Clin. Biochem. Nutr. 40 (3), 163 (2007). http://dx.doi.org/10.3164/jcbn.40.163 
7. M. S. Hossain, M. Asadujjaman, M. R. I. Khan, M. Ahmed and A. Islam, IJPSR 2 (2), 404 (2011). ISSN: 0975-8232

8. J. K. Grover, S. Yadav, V. Vats, J. Ethnopharmacol. 81 (1), 81(2002). http://dx.doi.org/10.1016/S0378-8741(02)00059-4

9. J. Stano, K. Mičieta, P. Kovács, K. Neubert, H. Tintemann and N. Tribulová, Acta Biotechnologica 21 (1), 83 (2002). http://dx.doi.org/10.1002/1521-3846(200102)21:1<83::AID-ABIO83>3.0.CO;2-7

10. A. Ghani, (LAP Lambert Academic Publishing, Eds. 2, 2003) pp. 193, 273 and 287.

11. J. P. Quanico, E. C. Amor and G. C. Perez, Philippine J. Sci. 137 (1), 69 (2008).

12. V. Sankari, V. Chitra, R. Jubilee, J. P. Silambu and D. Raju, Scholars Res. Lib. Der Pharmacia Lettre 2 (1), 291 (2010).

13. T. Satyanarayana, T. Sarita, M. Balaj, A. Ramesh and K. B Murthy, Saudi pharm. J., 12 (2-3), 108 (2003).

14. M. R. I. Khan, M. A. Islam, M. S. Hossain, M. Asadujjaman, M. I. I. Wahed, B. M. Rahman, A. S. M Anisuzzaman, S. M. Shaheen and M. Ahmed J. Sci. Res. 2 (1), 158 (2010). doi: $10.3329 /$ jsr.v2i1.2769

15. G. Sumana and S. A. Suryawanshi, Indian J. Exp. Biol. 39, 748 (2001).

16. Y. Srivastava, H. V. Bhatt, Y. Verma, K. Venkaiah and B. H. Raval, Phytother. Res. 7, 285 (1993). http://dx.doi.org/10.1002/ptr.2650070405

17. M. Mahomed and J. A. Ojewole, Exp. Clin. Pharmacol. 25, (2003).

18. A. Andrade-Cetto, and H. Wiedenfeld, J. Ethnopharmacol. 90, 217 (2004). http://dx.doi.org/10.1016/j.jep.2003.09.049

19. J. A. Ojewole, Exp Clin Pharmacol. 27 (8), 547 (2005).

20. A. Riyad, S. Abdul-Ghani, Abdul-Salam, and S. M. Suleiman, Planta. Med. 54, 286 (1988). http://dx.doi.org/10.1055/s-2006-962434

21. S. P. Tarfa, P. K. Joseph, and K.T. Augusti, Curr Sci. 57, 32 (1988).

22. S. R. Sharma, S. K. Dwivewdi, and D. Swarup, Ind. J. of Exp Biol. 34, 372 (1996).

23. J. K. Grover, V. Vats, and S. S. Rathi, J. Ethnopharmacol. 73 (3), 461 (2000). http://dx.doi.org/10.1016/S0378-8741(00)00319-6 